\title{
Target single- and double-spin asymmetries in DVCS off a longitudinal polarized hydrogen target at HERMES
}

\author{
David Mahon \\ University of Glasgow \\ E-mail: d.mahonephysics.qla.ac.ul

\section{Caroline RiedI} \\ DESY, Zeuthen \\ E-mail: criedlemail.desy.de
}

\begin{abstract}
We present recently-published HERMES asymmetry measurements for the hard exclusive leptoproduction of real photons from a longitudinally polarised hydrogen target using a $27.57 \mathrm{GeV}$ longitudinally polarised HERA positron beam. These asymmetries arise from the deeply virtual Compton scattering and Bethe-Heitler processes. Two asymmetries are simultaneously extracted in the azimuthal distribution of produced real photons about the direction of the exchanged photon: $A_{\mathrm{UL}}$ with respect to the polarisation of the target and $A_{\mathrm{LL}}$ with respect to the product of the beam and target polarisations. The results are presented in comparison with predictions from a generalised parton distribution model. The $\sin \phi$ and $\cos (0 \phi)$ modulations of the $A_{\mathrm{UL}}$ and $A_{\mathrm{LL}}$ respectively, are observed to be compatible with the predictions from this model. An unexpected $\sin (2 \phi)$ amplitude is also observed for $A_{\mathrm{UL}}$.
\end{abstract}

XVIII International Workshop on Deep-Inelastic Scattering and Related Subjects April 19 -23, 2010

Convitto della Calza, Firenze, Italy 


\section{Introduction}

Deeply Virtual Compton Scattering (DVCS) i.e. the hard exclusive leptoproduction of real photons, is regarded as the simplest interaction in which to access information on Generalised Parton Distributions (GPDs) [U, [2], an informational framework encompassing nucleon form factors and parton distribution functions as moments and limiting cases respectively. At leading twist (i.e. twist-2) and leading order, there are four quark spin $\frac{1}{2}$ GPDs (denoted $H, E, \widetilde{H}$ and $\widetilde{E}$ ) which provide a multi-dimensional description of the partonic structure of the nucleon.

The $e p \rightarrow e p \gamma$ process has two contributions to the cross section. One is from DVCS and the other is from Bethe-Heitler (BH) which is exactly calculable in the framework of QED and does not provide information about GPDs. At HERMES, the BH contribution dominates that from the DVCS process. However, useful information related to GPDs is accessible via the interference term $I$ in the scattering amplitude.

This GPD information is contained in Fourier coefficients from the expansion of the scattering amplitude in the azimuthal distribution of the real photons produced during the process [[]]. These amplitudes are functions of kinematic variables and Compton Form Factors (CFFs), denoted $\mathscr{H}$, $\mathscr{E}, \widetilde{\mathscr{H}}$ and $\widetilde{\mathscr{E}}$, which are convolutions of the corresponding GPD with a hard scattering kernel.

Information relating to these Fourier coefficients are extracted from experimental data by forming asymmetries with respect to the azimuthal angle $\phi$ between the lepton scattering and photoproduction planes.

The single-spin $A_{\mathrm{UL}}$ (double-spin $A_{\mathrm{LL}}$ ) asymmetry dependent on the longitudinal polarisation of the target (beam and target) is presented. These are written as

$$
\begin{aligned}
& A_{\mathrm{UL}}(\phi) \equiv \frac{\left[\sigma^{\leftarrow \Rightarrow}(\phi)+\sigma^{\rightarrow \Rightarrow}(\phi)\right]-\left[\sigma^{\leftarrow \Leftarrow}(\phi)+\sigma^{\rightarrow \Leftarrow}(\phi)\right]}{\left[\sigma^{\leftarrow \Rightarrow}(\phi)+\sigma^{\rightarrow \Rightarrow}(\phi)\right]+\left[\sigma^{\leftarrow \Leftarrow}(\phi)+\sigma^{\rightarrow \Leftarrow}(\phi)\right]} \\
& =\frac{K_{\mathrm{DVCS}} \sum_{n=1}^{2} s_{n, \mathrm{LP}}^{\mathrm{DVCS}} \sin (n \phi)-\frac{e_{\ell} K_{I}}{P_{1}(\phi) P_{2}(\phi)} \sum_{n=1}^{3} s_{n, \mathrm{LP}}^{I} \sin (n \phi)}{\frac{1}{P_{1}(\phi) P_{2}(\phi)}\left[K_{\mathrm{BH}} \sum_{n=0}^{2} c_{n, \mathrm{u}}^{\mathrm{BH}} \cos (n \phi)-e_{\ell} K_{I} \sum_{n=0}^{3} c_{n, \mathrm{u}}^{I} \cos (n \phi)\right]+K_{\mathrm{DVCS}} \sum_{n=0}^{2} c_{n, \mathrm{u}}^{\mathrm{DVCS}} \cos (n \phi)}, \\
& A_{\mathrm{LL}}(\phi) \equiv \frac{\left[\sigma^{\rightarrow \Rightarrow}(\phi)+\sigma^{\leftarrow \Leftarrow}(\phi)\right]-\left[\sigma^{\leftarrow \Rightarrow}(\phi)+\sigma^{\rightarrow \Leftarrow}(\phi)\right]}{\left[\sigma^{\rightarrow \Rightarrow}(\phi)+\sigma^{\leftarrow \Leftarrow}(\phi)\right]+\left[\sigma^{\leftarrow \Rightarrow}(\phi)+\sigma^{\rightarrow \Leftarrow}(\phi)\right]} \\
& =\frac{1}{P_{1}(\phi) P_{2}(\phi)}\left[K_{\mathrm{BH}} \sum_{n=0}^{1} c_{n, \mathrm{LP}}^{\mathrm{BH}} \cos (n \phi)-e_{\ell} K_{I} \sum_{n=0}^{2} c_{n, \mathrm{LP}}^{I} \cos (n \phi)\right]+K_{\mathrm{DVCS}} \sum_{n=0}^{1} c_{n, \mathrm{LP}}^{\mathrm{DVCS}} \cos (n \phi) \\
& \frac{1}{P_{1}(\phi) P_{2}(\phi)}\left[K_{\mathrm{BH}} \sum_{n=0}^{2} c_{n, \mathrm{u}}^{\mathrm{BH}} \cos (n \phi)-e_{\ell} K_{I} \sum_{n=0}^{3} c_{n, \mathrm{u}}^{I} \cos (n \phi)\right]+K_{\mathrm{DVCS}} \sum_{n=0}^{2} c_{n, \mathrm{u}}^{\mathrm{DVCS}} \cos (n \phi)
\end{aligned}
$$

where $\sigma$ denotes the $e p \rightarrow e p \gamma$ cross-section, $\rightarrow(\leftarrow)$ represents the beam helicity state parallel (anti-parallel) to the beam momentum, and $\Leftarrow(\Rightarrow)$ represents the target polarisation state parallel (anti-parallel) to the beam. The lepton propagators of the BH process are denoted $P_{1}(\phi)$ and $P_{2}(\phi)$. The $K$ terms are kinematic factors: $K_{\mathrm{BH}}=1 /\left(x_{\mathrm{B}}^{2} t\left(1+\varepsilon^{2}\right)^{2}\right), K_{\mathrm{DVCS}}=1 / Q^{2}$ and $K_{I}=1 /\left(x_{\mathrm{B}} y t\right)$, where $Q^{2}$ is the negative square of the virtual photon four-momentum, $t$ the squared momentum 


\begin{tabular}{|c||c|c|c|c|}
\hline $\begin{array}{c}\text { Asymmetry } \\
\text { Amplitude }\end{array}$ & $\begin{array}{c}\text { Contributory Fourier- } \\
\text { Coefficients }\end{array}$ & $\begin{array}{c}\text { Power of } \frac{1}{Q} \\
\text { Suppression }\end{array}$ & $\begin{array}{c}\text { Dominant CFF } \\
\text { Dependence }\end{array}$ & $\begin{array}{c}\text { Twist } \\
\text { Level }\end{array}$ \\
\hline \hline$A_{\mathrm{UL}}^{\sin \phi}$ & $s_{1, \mathrm{LP}}^{I}$ & 1 & $\operatorname{Im} \mathscr{C}_{\mathrm{LP}}^{I}$ & 2 \\
& $s_{1, \mathrm{LP}}^{\mathrm{DVCS}}$ & 2 & $\operatorname{Im} \mathscr{C}_{\mathrm{LP}}^{\mathrm{DVCS}}$ & 3 \\
\hline$A_{\mathrm{UL}}^{\sin (2 \phi)}$ & $s_{2, \mathrm{LP}}^{I}$ & 2 & $\operatorname{Im} \mathscr{C}_{\mathrm{LP}}^{I}$ & 3 \\
& $s_{2, \mathrm{LP}}^{\mathrm{DVCS}}$ & 2 & $\operatorname{Im} \mathscr{C}_{\mathrm{T}, \mathrm{LP}}^{\mathrm{DVCS}}$ & 2 \\
\hline$A_{\mathrm{UL}}^{\sin (3 \phi)}$ & $s_{3, \mathrm{LP}}^{I}$ & 1 & $\operatorname{Im} \mathscr{C}_{\mathrm{T}, \mathrm{LP}}^{I}$ & 2 \\
\hline \hline$A_{\mathrm{LL}}^{\cos (0 \phi)}$ & $c_{0, \mathrm{LP}}^{I}$ & 1 & $\operatorname{Re} \mathscr{C}_{\mathrm{LP}}^{I}$ & 2 \\
& $c_{0, \mathrm{LP}}^{\mathrm{DVCS}}$ & 1 & $\operatorname{Re} \mathscr{C}_{\mathrm{LP}}^{\mathrm{DVCS}}$ & 2 \\
\hline$A_{\mathrm{LL}}^{\cos \phi}$ & $c_{1, \mathrm{LP}}^{I}$ & 1 & $\operatorname{Re} \mathscr{C}_{\mathrm{LP}}^{I}$ & 2 \\
& $c_{1, \mathrm{LP}}^{\mathrm{DVCS}}$ & 3 & $\operatorname{Re} \mathscr{C}_{\mathrm{LP}}^{\mathrm{DVCS}}$ & 3 \\
\hline$A_{\mathrm{LL}}^{\cos (2 \phi)}$ & $c_{2, \mathrm{LP}}^{I}$ & 2 & $\operatorname{Re} \mathscr{C}_{\mathrm{LP}}^{I}$ & 3 \\
\hline
\end{tabular}

Table 1: The correspondences between the asymmetry amplitudes extracted from the data set and the CFFdependent Fourier coefficients of the $e p \rightarrow e p \gamma$ cross-section. The subscript $\mathrm{T}$ refers to $\mathscr{C}$-functions that involve leading-order gluon transversity CFFs which are further suppressed by $\frac{\alpha_{s}}{\pi}$.

transfer to the target, $y$ the fraction of the beam energy $E$ carried by the virtual photon in the target rest frame, $x_{\mathrm{B}}=\frac{Q^{2}}{2 M y E}$ and $\varepsilon \equiv 2 x_{\mathrm{B}} \frac{M}{Q}$, with $M$ representing the mass of the proton. In the case of positron scattering, the beam charge $e_{\ell}=+1$.

These asymmetries are simultaneously extracted using the maximum likelihood fitting formalism [四] from HERMES data taken on a longitudinally polarised hydrogen target using a $27.57 \mathrm{GeV}$ longitudinally polarised positon beam. They are decomposed as

$$
A_{\mathrm{UL}}(\phi) \simeq \sum_{1}^{3} A_{\mathrm{UL}}^{\sin (n \phi)}+A_{\mathrm{UL}}^{\cos (0 \phi)}, \quad A_{\mathrm{LL}}(\phi) \simeq \sum_{0}^{2} A_{\mathrm{LL}}^{\cos (n \phi)},
$$

where the asymmetry amplitudes relate to combinations of Fourier coefficients in the numerators of Eqs. 1.1 and 1.2 as outlined in Table 1. The $A_{\mathrm{UL}}^{\cos (0 \phi)}$ term was added as a test of the normalisation of the fitting procedure and was found to be zero as expected. The Fourier coefficients from the interference (squared-DVCS) terms are related to a linear (bilinear) combination of CFFs, at differing levels of suppression. This relationship is encoded in $\mathscr{C}$-functions.

Information can be accessed on the imaginary (real) part of $\mathrm{CFF} \mathscr{C}_{\mathrm{LP}}^{I}$, expressed as [B]]

$$
\mathscr{C}_{\mathrm{LP}}^{I}=\frac{x_{\mathrm{B}}}{2-x_{\mathrm{B}}}\left(F_{1}+F_{2}\right)\left(\mathscr{H}+\frac{x_{\mathrm{B}}}{2} \mathscr{E}\right)+F_{1} \widetilde{\mathscr{H}}-\frac{x_{\mathrm{B}}}{2-x_{\mathrm{B}}}\left(\frac{x_{\mathrm{B}}}{2} F_{1}+\frac{t}{4 M^{2}} F_{2}\right) \widetilde{\mathscr{E}}
$$

via the $A_{\mathrm{UL}}^{\sin \phi}\left(A_{\mathrm{LL}}^{\cos \phi}\right)$ amplitude which enters at leading-twist level via the interference term, with a suppressed twist -3 contribution from the squared-DVCS term. At HERMES kinematics, the dominant CFF is the quark-helicity dependent distribution $\widetilde{\mathscr{H}}$.

\section{Results}

Recently-published HERMES measurements [15, 囵] of the $A_{\mathrm{UL}}^{\sin (n \phi)}$ and $A_{\mathrm{LL}}^{\cos (n \phi)}$ amplitudes are shown (for $n \leq 2$ ) in Figs. 1 and 2 respectively, in comparison with theoretical predictions from 


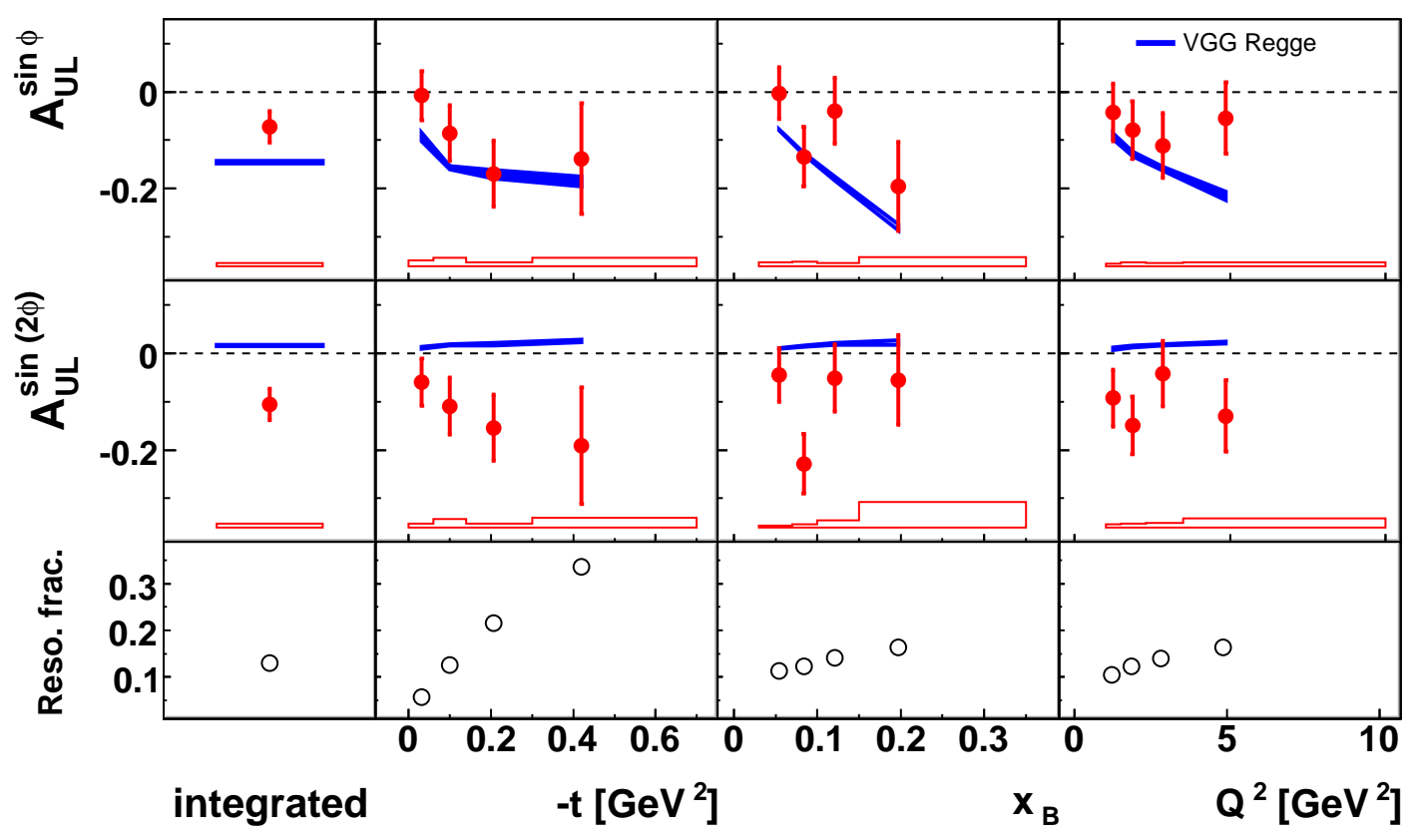

Figure 1: Amplitudes of the single-spin target asymmetry $A_{\mathrm{UL}}$ sensitive to the interference and squaredDVCS terms, for positrons incident on a longitudinally polarised hydrogen target, as a function of $-t, x_{\mathrm{B}}$, and $Q^{2}$. The integrated column shows the asymmetry values when the extraction is performed in a single bin across the entire kinematic range of the data set. The error bars (open bands) show the statistical (systematic) uncertainties and the solid bands represent the predictions from the VGG Regge GPD model [ $[$ ]]. There is an additional $4.2 \%$ scale uncertainty due to the precision of the measurement of the target polarisation. The estimated fractional contributions from resonance production are presented in the bottom panel.

the GPD model outlined in Ref [四]. The leading-twist $A_{\mathrm{UL}}^{\sin \phi}$ amplitude is observed to be non-zero when integrated over the entire data set and in agreement with the predicted kinematic trends. The $A_{\mathrm{UL}}^{\sin (2 \phi)}$ amplitude is unexpectedly large and of opposite sign than predicted. This may arise from the unknown contribution from leading-order gluon transversity CFFs appearing in $\mathscr{C}_{\mathrm{T}, \mathrm{LP}}^{\mathrm{DVCS}}$. The $A_{\mathrm{UL}}^{\sin (3 \phi)}$ (not shown) relates solely to gluon GPDs via $I$ and is consistent with zero across the plotted kinematic range.

The $A_{\mathrm{LL}}^{\cos (0 \phi)}$ and $A_{\mathrm{LL}}^{\cos \phi}$ amplitudes are unlike others presented here in that they also receive dominant contributions from also the squared-BH term. They both receive leading-twist contributions from the interference term with the $\cos (0 \phi)(\cos \phi)$ harmonic also receiving a twist -2 (twist-3) contribution from the squared-DVCS term. The $A_{\mathrm{LL}}^{\cos (0 \phi)}$ is found to be in agreement with theoretical predictions of a non-zero amplitude. The other two amplitudes are consistent with zero as expected. From the statistical precision however, no kinematic dependences can be discerned for this asymmetry.

\section{Acknowledgments}

I gratefully acknowledge the HERMES management for inviting me to give this talk, and Caroline Riedl who kindly stepped in to present it after Eyjafjallajokull disrupted my travel plans. 


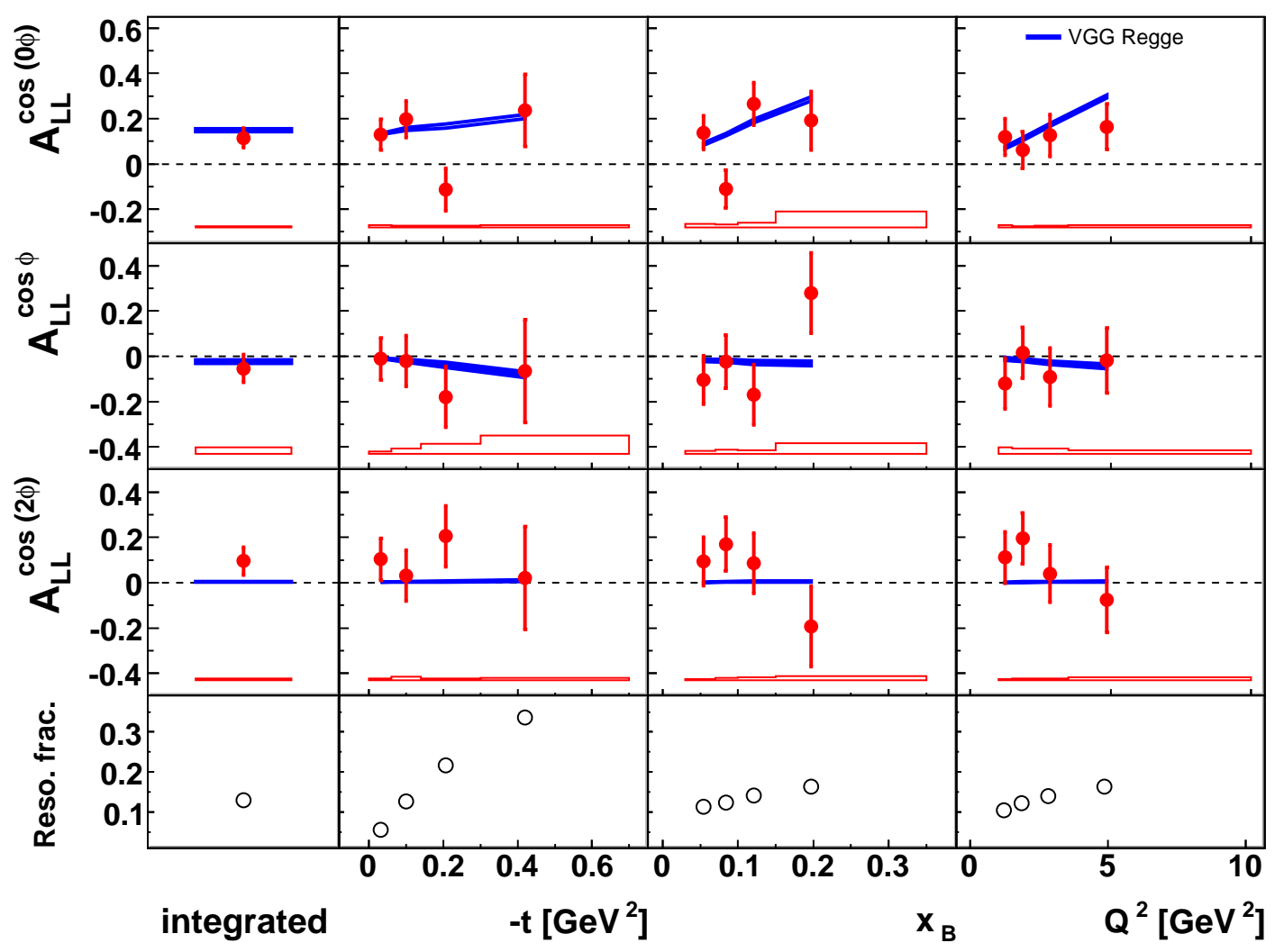

Figure 2: Amplitudes of the double-spin asymmetry $A_{\mathrm{LL}}$ sensitive to the interference, squared-DVCS and squared-BH terms, for positrons incident on a longitudinally polarised hydrogen target, as a function of $-t$, $x_{\mathrm{B}}$, and $Q^{2}$. The labelling of the figure is the same as in Fig. 1 with the exception of a 5.3\% scale uncertainty due to the precision of the measurements of the beam and target polarisations.

Thanks also to the University of Glasgow for their financial support.

\section{References}

[1] F. M. Dittes, et al., Phys. Lett. B209, 325-329 (1988).

[2] D. Mueller, et al., Fortschritte der Physik 42, 101 (1994).

[3] A. Belitsky, D. Muller and A. Kirchner, Nucl. Phys. B 629, 323 (2002).

[4] F. James, MINUIT, D507, CERN (1978).

[5] A. Airapetian et al., JHEP 06 (2010) 019.

[6] D. Mahon, Ph.D. thesis, University of Glasgow, DESY-THESIS-2010-023 (2010).

[7] M. Vanderhaeghen, P.A.M. Guichon, M. Guidal, Phys. Rev. D60, 094017 (1999). 